\title{
Going against the flow
}

\section{Ion transport: the division between active transporters and passive channels is beginning to blur}

\section{$\mathrm{T}$}

Louis J. DeFelice o function properly, living cells must maintain ions and small molecules at concentrations that are far different from those in their local environments. The electrochemical gradients formed in this way can be used by cells as a source of energy to drive other processes, for example membrane transport or the generation of electrical signals in specialized cells - such as those in nerves and muscle. Internal sodium ions are kept at roughly one-tenth of their external concentration, and the reverse is true for potassium ions. These ion gradients are maintained by a transmembrane protein (the sodium-potassium pump, an enzymic transporter), which actively pumps out sodium ions and pumps in potassium ions. Then, as an electrical signal (action potential) propagates along a nerve cell, sodium and potassium channels open, allowing the rapid flow of sodium ions into and potassium ions out of the cell. From many such examples, the concept emerges that transporters generate ionic gradients and channels dissipate them: transporters and channels seem as different as oil and water.

But this distinction depends on what we classify as 'transporter'. For enzymatic transporters, the difference seems clear - they use chemical energy to generate gradients. But for another class of transporters, known as co-transporters, the distinction is more difficult. Co-transporters can also create gradients, but they do so by using the energy stored in ionic gradients established by pumps - and are thus secondarily active. For example, co-transporters would use the sodium gradient set up by the sodium pump to power the transport of another substrate, such as a neurotransmitter, against its own gradient. However, researchers tend to model co-transporters as if they were enzymes, even though they have authentic channel properties. What are the origins of this riddle and how can it be resolved?

Channels and transporters did not originate as separate entities. Alan Hodgkin and colleagues unravelled the ionic basis of action potentials in the 1940s. But to explain sodium and potassium permeability they initially postulated transporters, not channels - ions were carried across the membrane rather than moving through a pore. Soon afterwards, prominently in the work of Hodgkin and Richard Keynes, the notion of electrodiffusion through narrow pores (channels) emerged. Concurrently,

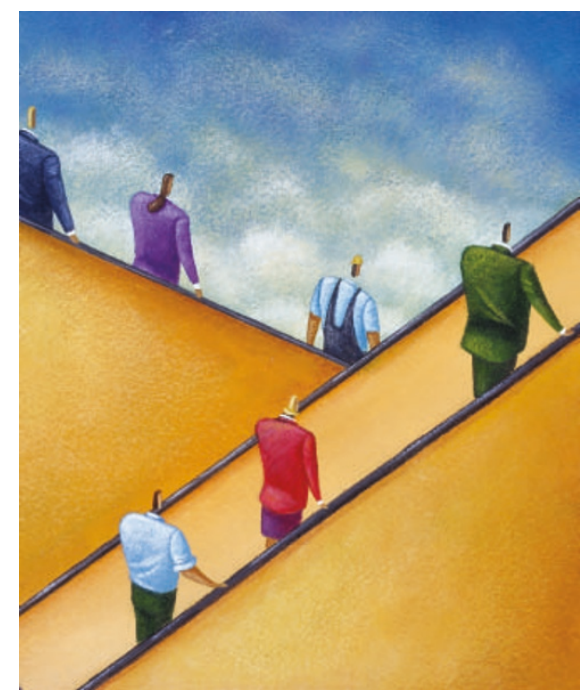

researchers struggled with electrodiffusion as a means to concentrate metabolites 'against the gradient.' Wilfred F. Widdas, in particular, introduced the harbinger of co-transport, in which the gradient of one species drives the transport of another. The adoption of enzymatic theory and carrier kinetics for co-transporters soon followed, and two camps evolved: electrodiffusion theory governed channels, and enzyme theory governed transporters. Different methodologies contributed to this separation, as channel biophysicists relied mainly on electrical measurements, whereas transport physiologists preferred radiolabelled uptake experiments. Indeed, early attempts at an electrical description of active transport were disappointing because of the comparatively low signal.

As a result, the study of transporters (including co-transporters) and the study of channels grew apart. But in the past decade the cloning of co-transporters, combined with the measurement of tiny currents through individual channel and transporter proteins by high-resolution electrophysiology (patch clamp) has begun to bridge this historical gap. Almost every co-transporter studied in this way exhibits ion channel properties. Glutamate and dopamine co-transporters harbour chloride-selective channels. GABA, serotonin and norepinephrine co-transporters contain sodium and lithium channels. Recently, a presumed chloride channel has been shown to be a co-transporter, but a simple mutation returns it to pure chloride selectivity. In another case, an iron co-transporter naturally mutates into a calcium channel. We may expect many other such examples as the application of ion channel techniques are applied to transporters. Even the sodium-potassium pump has been shown to have ion-channel properties under special conditions.

The challenge now is to reconcile the signature property of a co-transporter - the ability to accumulate substrates 'against the gradient' - with its nature as an ion channel. At least two possibilities arise. Co-transporters might obey enzyme theory when they are active, but occasionally slip into a passive channel mode. Alternatively, co-transporters might obey channel theory and rely on flux coupling for their secondary activity.

In flux coupling, an ionic current drives the secondary transport of a substrate. This may occur because a channel is too narrow to allow the ion and substrate to pass one another and so the powerful flow of the ion down its gradient carries the substrate along against its own gradient (single file diffusion). Electrical and osmotic forces can also drive flux coupling - for example, an ionic current can drive water against its own osmotic gradient and vice versa. On a cautionary note, flux coupling in the same direction seems simple and obvious, but a much knottier problem is that of counter flow, in which one species moves in the opposite direction to the other and yet coupling is still positive.

We seem closer than ever to understanding similarities between these seemingly disparate membrane proteins, due to the advent of structures for several ion-selective channels. These include a chloride-hydrogen co-transporter (first presumed to be a chloride channel), lactose and glutamate co-transporters and an extremely highresolution solution for an ammonia channel (once thought to be a co-transporter). Releasing co-transporters from the grip of enzyme theory would give us a fresh start on mechanisms that would include all transport properties, and lead to a possible reunion of co-transporters and channels.

Louis J. DeFelice is in the Department of Pharmacology and the Center for Molecular Neuroscience at Vanderbilt University Medical Center in Nashville, Tennessee 37232, USA.

\section{FURTHER READING}

DeFelice, L. J. Trends Neurosci. 27, 352-359 (2004). Hodgkin, A. L. Chance and Design (Cambridge Univ. Press, 1992).

Lauger P. Electrogenic Ion Pumps (Sinauer Associates, 1991).

Stein, W. D. Channels, Carriers, and Pumps (Academic Press, New York, 1990). 